\title{
An analysis of docking study on tuberculosis inhibitors
}

\author{
Anuja Rangi ${ }^{1}$, Saroj Rani ${ }^{2}$, Savita Kumari ${ }^{3}$, Sudhir Kumar ${ }^{3}$ and Manoj Giri ${ }^{4 \star}$ \\ ${ }^{1}$ Department of Biotechnology, University Institute of Engineering \& Technology Kurukshetra -136 119, Haryana, India \\ ${ }^{2}$ Department of Biotechnology, Seth Jai Prakash Mukund Lal Institute of Engineering \& Technology, Radaur- 135133 , \\ Haryana, India \\ ${ }^{3}$ Department of Bioinformatics, Chaudhary Charan Singh Haryana Agricultural University, Hisar- 125 004, Haryana, India \\ ${ }^{4}$ Departmnet of Applied Sciences, Haryana College of Technology \& Management, Kaithal-136 027, Haryana, India, \\ manojgiri1@rediffmail.com, 097295-73419, Fax No. (01746) 280711
}

\begin{abstract}
In the present paper nine molecules has been docked in the Acyl-CoA carboxylase carboxyltransferase domain 5 (AccD5) at GLY-193, GLY-194, GLY-434, ALA-435 pocket with very low energies. Six binding site on the crystallographic structure of AccD5 are examined with the structure-based design program DOCK. The active compounds found are polycyclic (aromatic as well as aliphatic) and nitrogen heterocyclic. Each molecule gave a good number of conformations showing the flexible behavior of the ligand. The total energy of receptor-ligand complexes has also been calculated.

Keywords: Docking, AccD5, TB, Vanderwall \& electrostatic energy.
\end{abstract}

\section{Introduction}

Tuberculosis abbreviated as TB for tubercle bacillus is one of the most common and deadly infectious disease caused by mycobacteria, mainly Mycobacterium tuberculosis. About 32\% of the world's population is infected to TB [1]. Myobacterium tuberculosis has two features that render it the deadliest infectious disease to date, its high infectivity (virulence) and its ability to enter latency for subsequent reactivation, a phenomenon that leads to a deadly synergy with AIDS [2-4]. In M. tuberculosis, Acyl-CoA carboxylase carboxyltransferase (ACCase) consists of three subunits namely $\alpha, \beta$ and $\varepsilon$ [5]. In molecular modeling, docking is a method which predicts the preferred orientation of one molecule to a second when bound to each other to form a stable complex. Knowledge of the preferred orientation in turn may be used to predict the strength of association or binding affinity between two molecules using for example scoring functions [6-7]. The protein structure and a database of potential ligands serve as inputs to a docking program. The success of a docking program depends on two components, the search algorithm and the scoring function [8]. Molecular docking algorithms fit molecules together in complementary fashions. The technique has attracted increasing attention as a way to predict the geometries of bimolecular complexes [9]. Most of docking programs in use account for a flexible ligand, and several are attempting to model a flexible protein receptor. Each snapshot of the pair is referred to as a pose [10]. Stochastic search, incremental construction, and multi-conformer docking are three ways to classify these algorithms. Auto Dock, Internal Coordinate Mechanics (ICM), Genetic Optimization for Ligand Docking (GOLD) etc are the representatives for stochastic search algorithm. These algorithms are based on genetic algorithms and Monte Carlo-simulated annealing $[11,12]$. After several decades of neglect, tuberculosis is receiving the increased attention that this global public health problem deserves. Although most of these new resources are being appropriately invested in TB control programs in countries where the TB epidemic is most severe, a significant commitment also is being made to basic research and the development of new diagnostic, treatment, and prevention tools, including new TB drugs [13]. The present study has been carried out to screen National Cancer Institute diversity database of compounds against tuberculosis (AccD5) using DOCK with the objective to find potential drug targets against AccD5.

\footnotetext{
Methodology

Software used: Chimera, DMS and DOCK software are used in the present study (http://www.csb.yale.edu/underguides/graphics/m idas/man/dms.html),(http://dock.compbio.ucsf.ed u/DOCK_5/index.html), (http://blaster.docking.org/zinc/).

Procedure: $3-D$ structure of AccD5 of mycobacterium tuberculosis was taken from Protein Data Bank (pdb id-2A7S). The active site was identified. From six chains only two chains A $\& D$ were used because the active sites were present at the interfaces of the chains. The procedure used for the study is follows as:

1. The receptor is first trimmed for the ligands i.e., all water molecules pertaining to the receptor molecule are removed.

2. The polar hydrogen's are added to the receptor.

3. Partial charges for the molecule with AMBER force field has been calculated and the energy is minimized using the information from the partial charge calculations [14].

4. dms Program is used and molecular surface data for sphere generation for docking was created.

5. The generated file from the dms program is used as one of the inputs of the INSPH file. The
} 
INSPH file is used for the input to SPHGEN program in DOCK.

6. Co-ordinates from the crystal structure of ligand are used to select the relevant spheres for grid and docking computations.

7. Sphere_Selector in DOCK is used to select the spheres.

8. The grid is generated using the GRID program in DOCK with input in grid.

9. The next step involved the docking of the receptor with the multiple ligand library using the DOCK program taking the input in dock.

\section{Conclusion of first round screening}

After the crucial steps of receptor preparation, we docked the receptor $2 \mathrm{~A} 7 \mathrm{~S}$ with $\mathrm{NCl}$ database $(1,27,000)$ then got 50 hits of chemical compound which fitted at AccD5's active site GLY 193, GLY 194, GLY 434, ALA 435 in first round of screening. Then, in the second round of screening, by changing the parameters of dock, 50 hits of first round are used as database for docking which give 9 hits. These are refinement of first round's result as shown with the "Figs (19)".

\section{Results and discussions}

A total of 1, 27,000 candidates (ligands) from $\mathrm{NCl}$ diversity database are used in the study. Six binding sites on the crystallographic structure of AccD5 has been examined with the structurebased design program DOCK 6.1. DOCK is used to filter the database of compounds and identify candidate ligands, which were complementary to one of the sites. The ligands are screened for their ability to dock within the catalytic traid of AccD5 and to find those chemical compounds which can inhibit the activity of AccD5 and for this two rounds of virtual screening are performed and finally got 9 molecules that bound at different binding pockets, mainly at GLY 193, GLY 194, GLY 434, ALA 435. These molecules has multiple conformations. The molecular surfaces are generated which are used to cluster spheres. Site points are constructed within $10 \AA$ of the ligand using the sphere generation accessory program of DOCK. The cluster of site points which occupied the binding site of the actual ligand in the crystal complex is selected from the three clusters obtained using SPHGEN utility of DOCK software, first cluster is selected having six spheres using sphere selector utility and box is generated around active sites as shown in "Fig. (10)". The scoring grid is generated with $5,789,846$ grid points as shown in "Fig. 10, 11 and 12 ". The final dock output shows the docking of nine compounds from the ligand library. Multiple low energy conformations of each inhibitor are generated using a simple molecular dynamics and minimization protocol and a unique set of conformations is collected. The selected conformations covered the range of energies of the entire set from the molecular dynamics calculations. The number of unique conformations generated for each ligand varied enormously, and gave some perception of the flexibility of the different inhibitor. It is expected that the specific geometry of each pocket would provide filtering of the large set of structures used as input to yield a relatively small and characteristic set of compounds and conformations that would allow the interpretation of the ligand and Non-nucleoside inhibitor-binding pocket (NNIBP) conformational features that gave rise to the binding mode of each docked compound as shown in "Fig. 13". Application of force field to the database proved to be more promising, reducing the number of docked conformations significantly. Inspection of the highest scoring inhibitor conformations, and their relative orientations, after docking of the database, showed only limited specificity for the inhibitor.

\section{References}

[1] Ishikawa N. Kekkaku (2005), 80(2), 89-94 [Pub Med].

[2] Bates I., Fenton C., Gruber J, Lalloo D., Lara A. M., Squire S. B., Theobald S., Thomson R. and Tolhurst R. (2004), Lancet Infect. Dis., 4, 368-375.

[3] Takayama K., Wang C. and Besra G. S. (2005), Clin. Micro. Rev., 18, 81-101.

[4] Ting-Wan Lin, Melrose M Melgar, Kurth Daniel and Swamidass S. Joshua (2005), 164-189.

[5] Diacovich L., Mitchell D., Pham H., Gago G., Melgar M. M., Khosla C, Gramajo H. and Tsai S.C. (2004), Biochemistry, 43, 14027-36;

[6] Ewing T., Makino S., Skillman A. and Kuntz I. (2001) J Comput Aided Mol. Des., 15 (5), 411-28.

[7] Bursulaya B., Totrov M., Abagyan R. and Brooks C (2003) J Comput Aided Mol Des., 17, 75563.

[8] Kitchen D. B., Decornez H, Furr J. R. and Bajorath J. (2004), Nature reviews, Drug discovery, 3 (11), 935-49.

[9] Irawin D. Kuntz, Elaine C. Meng and Brain K. Shoichet (1994), Acc. Chem. Res., 27 (5), 117-123.

[10] Shoichet B.K., Kuntz I. D. and Bodian D.L. (2004), Journal of Computational Chemistry, $13(3), 380-397$.

[11] Vose M. D. and Wright A. H. (1998), The simple genetic algorithm and the Walsh transform, Part I, Theory, Evolutionary Computation, 6(3), 253-273.

[12] J. De Vicente, J. Lanchares and R. Hermida (2003), Physics Letters A, 317.

[13] Zhang H., Yang Z., Shen Y. and Tong L. (2003), Science, 299, 2064-2067.

[14] Wang J.,Wang W., Kollman P. A. and Case D.A.(2006), Journal of Molecular Graphics \& Modeling, 25, 247-260. 


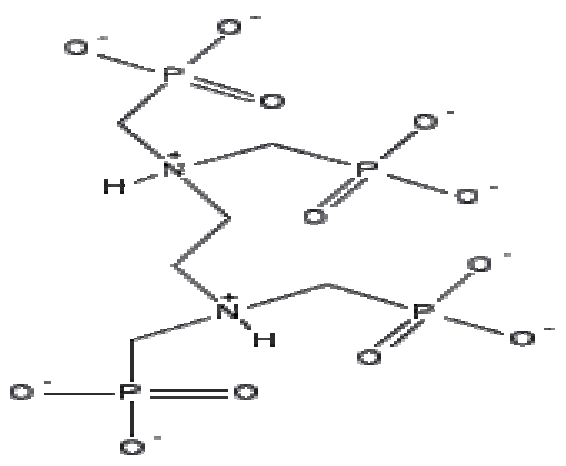

Fig. 1- Molecular structure of [bis (phosphonomethyl) amino] ethyl-(phosphonomethyl) amino] methylphosphonic acid with Grid score: -65.145767, Vander wall energy: -39.844387, Electrostatic energy: 25.30138

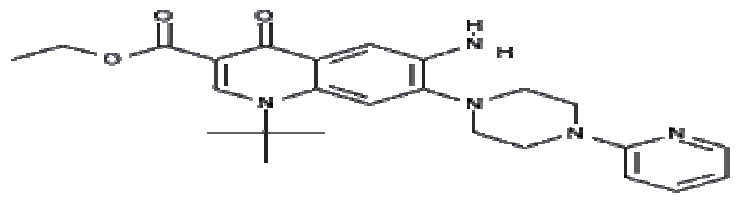

Fig. 2- Molecular structure of ethyl 6-amino-1-tert-butyl-4-oxo-7-(4-pyridin-2-ylpiperazin-1-yl) quinoline-3carboxylate with Grid score: -60.625904, Vander wall energy:- 61.079876, Electrostatic energy: 0.453971

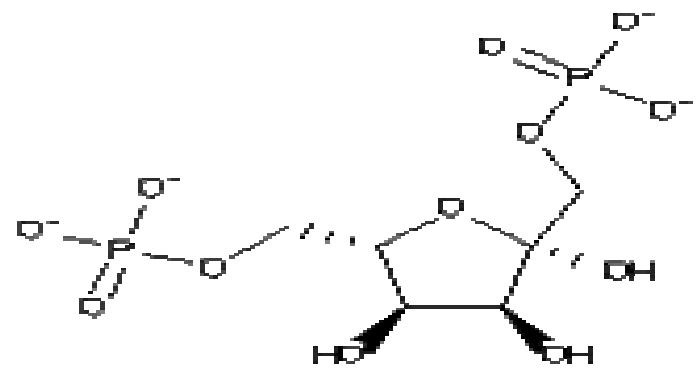

Fig. 3- Molecular structure of 2, 3, 4-trihydroxy-5-(phosphonooxymethyl) tetrahydrofuran-2-yl] methoxyphosphonic acid with Grid score: -58.104099 , Vander wall energy: -33.324596 , Electrostatic energy: -24.779503

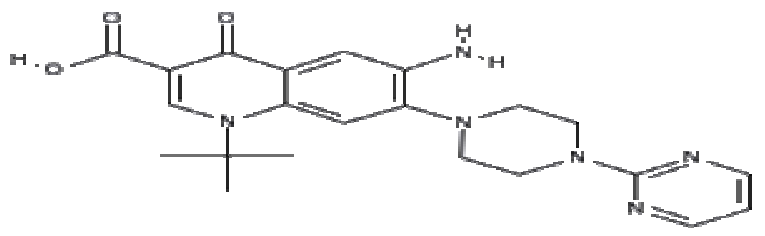

Fig.4 - Molecular structure of 6-Amino-1-(tert-butyl)-4-oxo-7-(4-pyrimidin-2-ylpiperazinyl) hydroquinoline-3carboxylic acid with Grid score: -57.046413 , Vander wall energy: -57.385811 , Electrostatic energy: 0.339396 


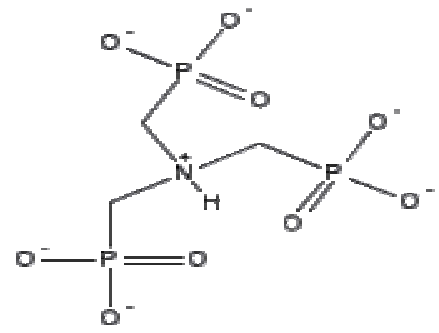

Fig. 5- Molecular structute of tris(phosphonatomethyl)azanium with Grid score: $\quad-56.337387$, Vander wall energy: -29.110706, Electrostatic energy: -27.226681<smiles>Nc1ccn(C[C@H](CO)C[C@@H](c2ccccc2)P(=O)([O-])[O-])c(=O)n1</smiles>

Fig. 6- Molecular structure of 3-[(4-amino-2-oxo-pyrimidin-1-yl) methyl]-4-hydroxy-1-phenyl-butyl]phosphonic acid with Grid score: -55.979668 , Vander wall energy: $\quad$-38.203125, Electrostatic energy: -17.776545.

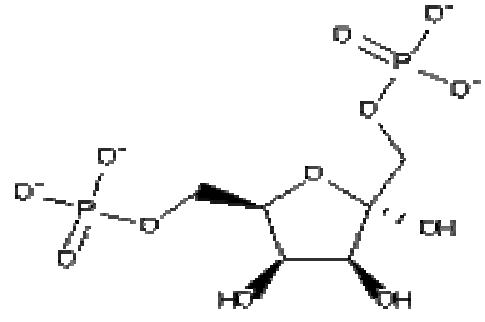

Fig. 7- Molecular structure of 2, 3, 4-trihydroxy-5-(phosphonooxymethyl) tetrahydrofuran-2-yl] methoxyphosphonic acid with Grid score: -55.662514 , Vander wall energy: -31.133688 , Electrostatic energy: -24.528826

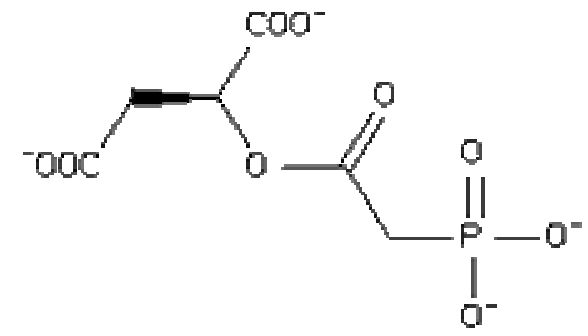

Fig. 8- Molecular structure of 2-(2-phosphonoacetyl) oxybutanedioic acid with Grid score: -55.544464, Vander wall energy: 29.976547, Electrostatic energy: -25.567919 


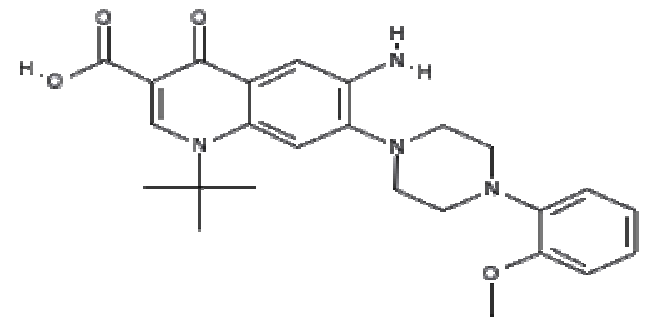

Fig. 9- Molecular structure of 6-Amino-1-(tert-butyl)-7-[4-(2-methoxyphenyl) piperazinyl]-4 oxohydroquinoline-3-carboxylic acid with Grid score: -55.192966, Vander wall energy: -54.840630, Electrostatic energy: -0.352338

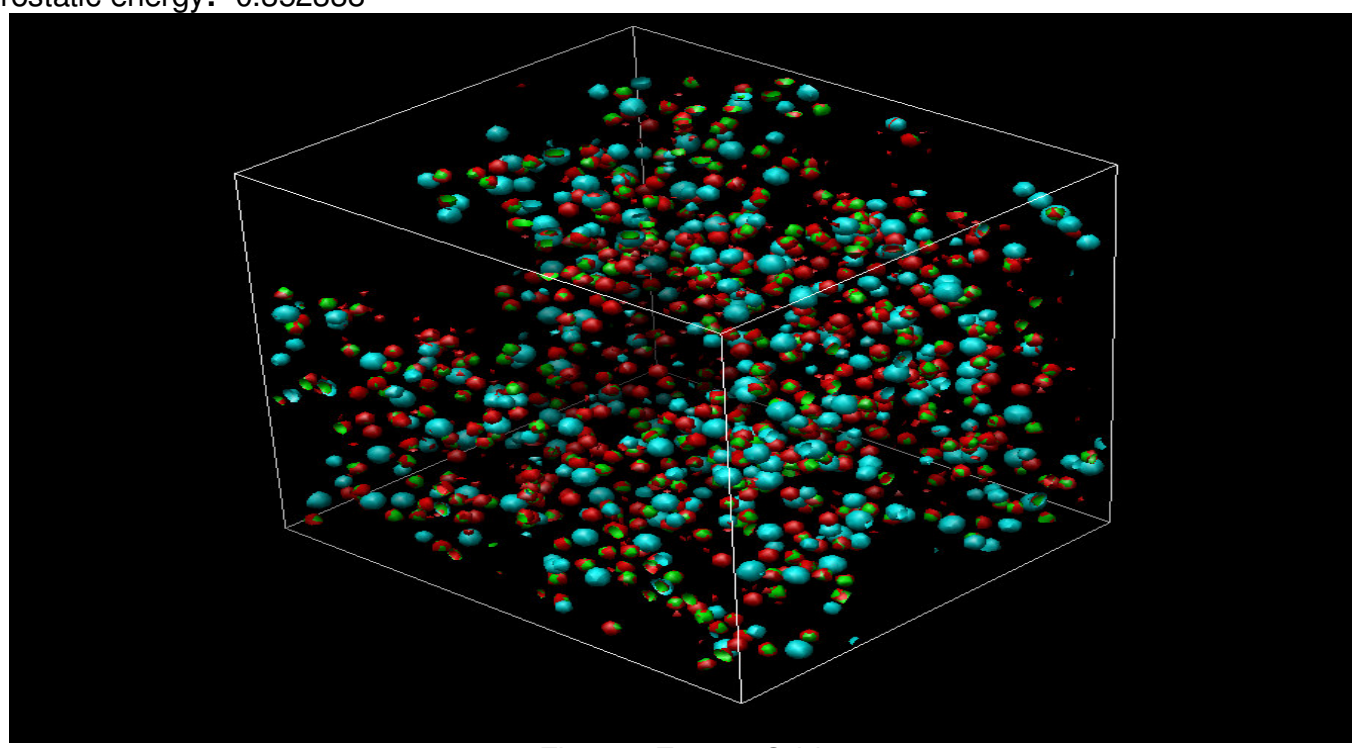

Fig. 10- Energy Grid

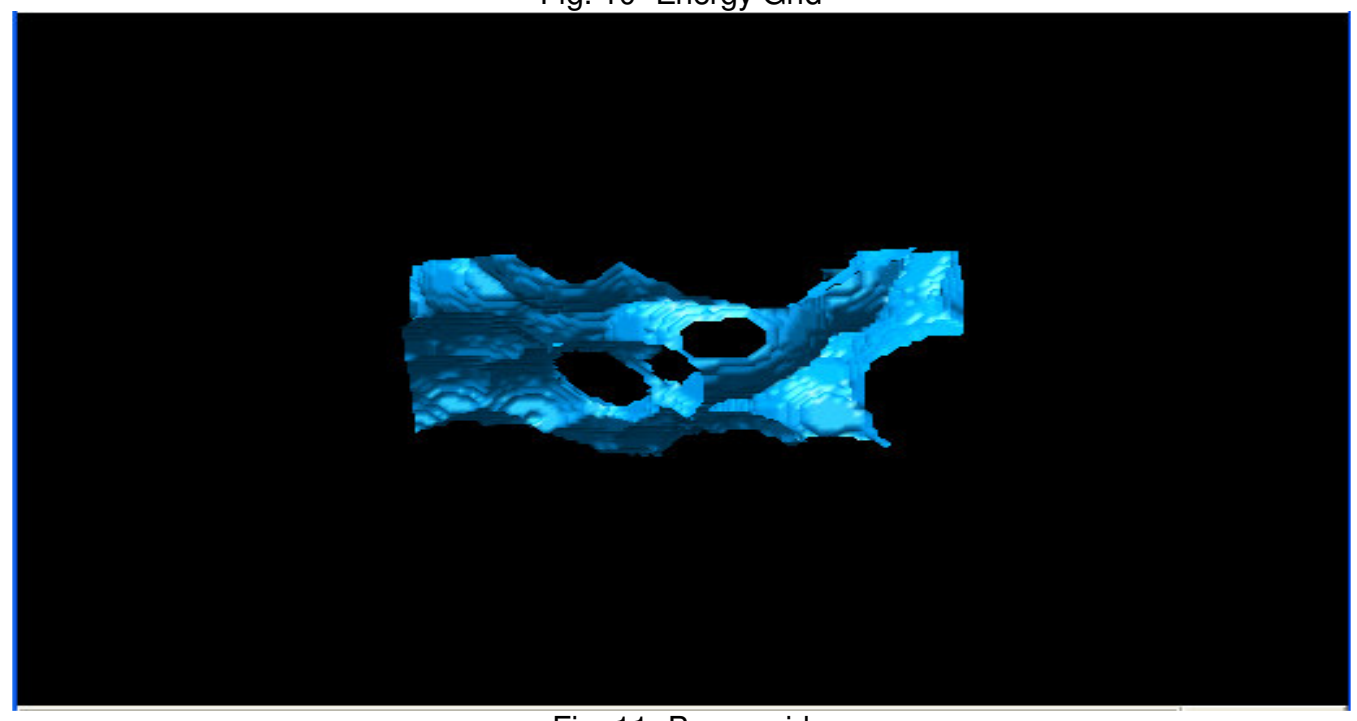

Fig. 11- Bump grid 

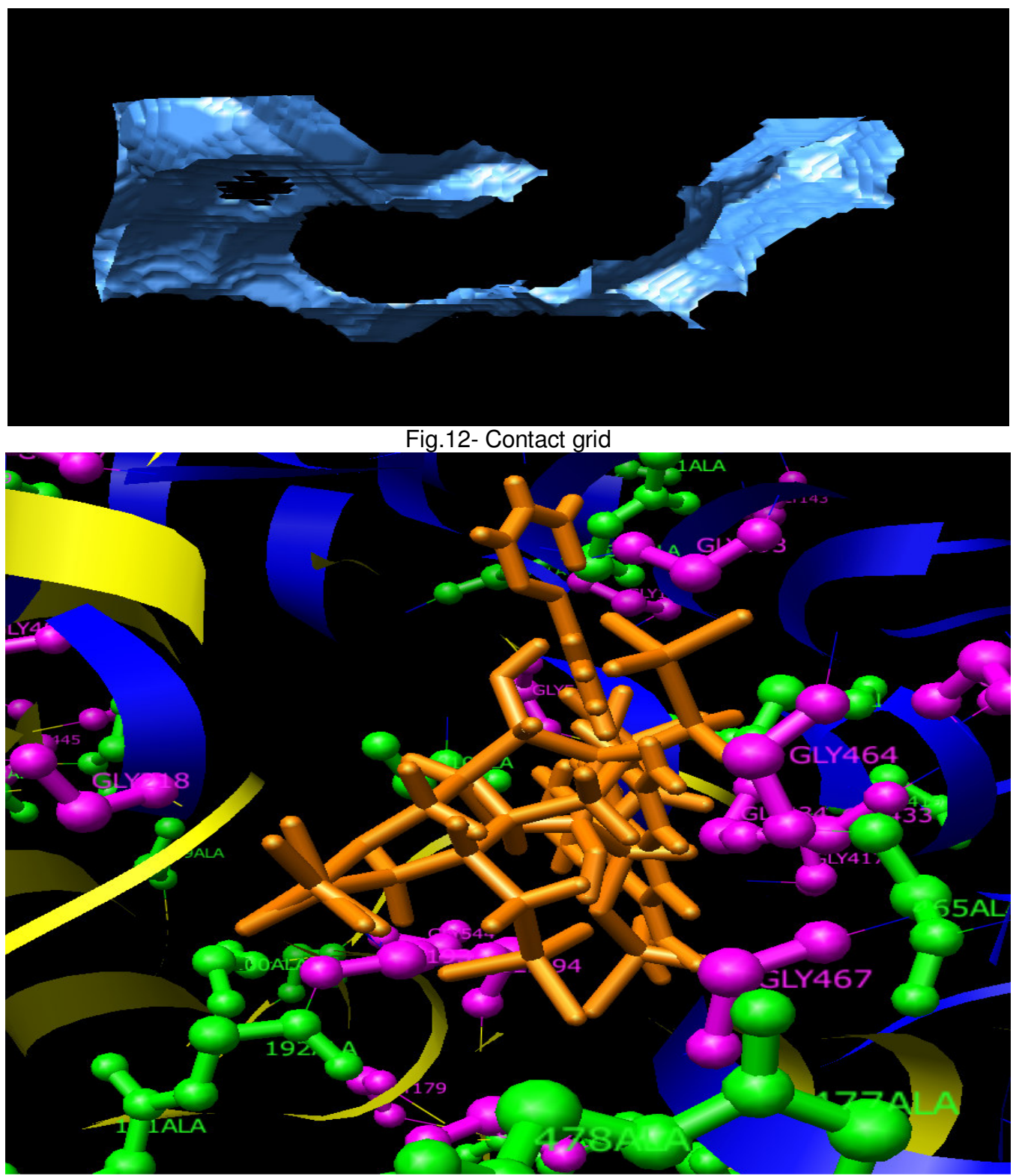

Fig.13- Molecule docked at AccD5‘s GLY 193, GLY 194, GLY 434, ALA 435 